\title{
Optimal Distance between the Termini of Stem P3 and P4 in Kin.46 Kinase Ribozyme
}

\author{
Bongrae Cho \\ Department of Applied Chemistry, Cheongju University, Cheongju 360-764, Korea.E-mail: brcho@cju.ac.kr \\ Received March 31, 2010, Accepted April 16, 2010
}

Key Words: Kinase ribozyme, Thiophosphorylation, [( $N$-Acryloylamino $)$ phenyl $]$ mercuric chloride, Firstorder rate

The Kin.46 kinase ribozyme catalyzes the transfer of the thiophosphate from ATP- $\gamma-\mathrm{S}$ to its own 5' hydroxyl end in the presence of oligonucleotide effector which is complementary to its 3' primer binding sequence (PBS) used in the amplification steps during the original selection for activity. ${ }^{1,2}$ Omitting the oligonucleotides reduces the observed catalytic rate constant $\left(k_{\text {obs }}\right)$ by $10^{3}$ to $10^{6}$-fold and addition of the deoxyoligonucleotide effector is necessary for its activity. The activator helix formed by the PBS and the oligo effector is connected by a 5nt "linker" region, $\mathrm{J} 1 / 4$, to the substrate-binding internal guide sequence, stem $\mathrm{P} 1$ and stabilizes the active conformation of the ribozyme by stabilizing the interaction between the linker, J1/4 and complementary nucleotides, J3/2, within the active site (Fig. 1)., If the single-stranded region $\mathrm{J} 2 / 3$ and $\mathrm{J} 3 / 2$ form part of the active site, potentially forming the ATP-binding element, and
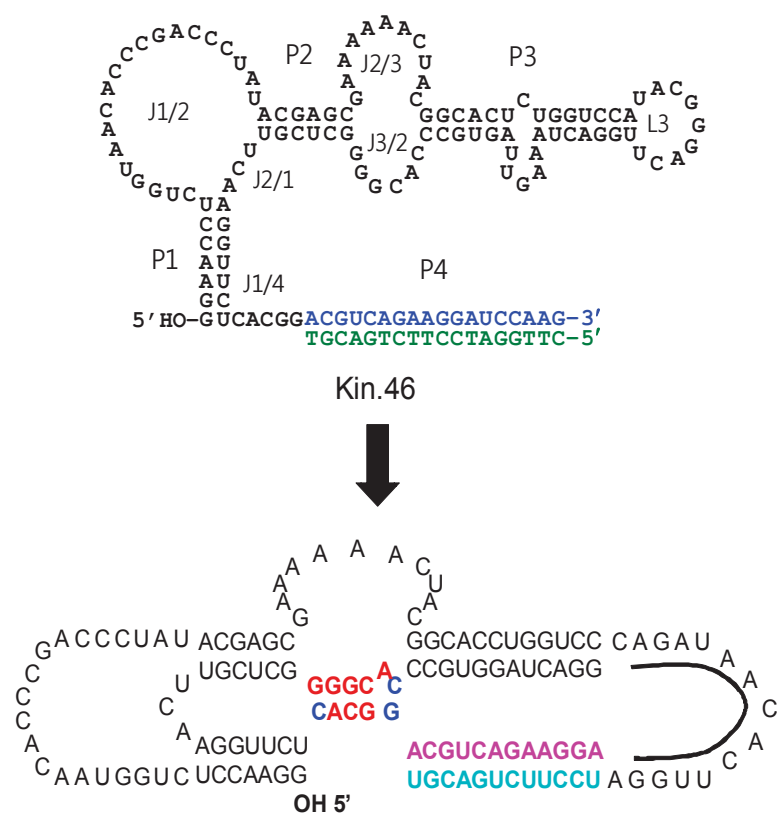

tethered ribozyme

Figure 1. Secondary structure models of Kin.46 ribozyme and the tethered ribozyme derived from Kin.46 (15-nt tether shown). Paired elements $\mathrm{P} 1$ through $\mathrm{P} 4$, connecting loop L3, and joining elements $\mathrm{J} 1 / 2, \mathrm{~J} 2 / 3, \mathrm{~J} 3 / 2, \mathrm{~J} 2 / 1$ and $\mathrm{J} 1 / 4$ are indicated. The tethered ribozyme is built from the two strands, outer strand and inner strand. Thick line in the tethered ribozyme indicates 13-nt DNA oligonucleotide complementary to the 15 -nt tether. the $5^{\prime} \mathrm{OH}$ acceptor is brought into this active site, then it follows that the proximal termini of $\mathrm{P} 3$ and $\mathrm{P} 4$ must be close to each other in space. In this work, to assess the overall architecture of the kinase ribozyme, especially an optimal distance of the termini of P3 and P4, several tethered species were generated by changing the connectivity of the stems P3 and P4 within the Kin.46 ribozyme and their activities were compared with [( $N$-acryloylamino $)$ phenyl $]$ mercuric chloride-polyacrylamide gel electrophoresis (APM-PAGE).

In the simplified variant derived from the original Kin.46 ribozyme, stem P3 separated by a small asymmetric loop into two paired segments was simplified by removing the loop and replacing P3 with 12 base-paired nucleotides (Fig. 1). The activator helix, P4 with 18 base-paired nucleotides was replaced with 12 base-paired nucleotides because the number (12-18) of nucleotides (nts) in P4 did not affect the ribozyme activity. We have observed that RNA and DNA effector strands are essentially equivalent in terms of their ability to activate catalysis. The resulting "tethered" ribozyme connected the termini of helices P3 and P4 with a single-stranded loop and was assembled in two pieces from an 83-nt "outer-RNA" and a 51-nt "inner-RNA". In this arrangement, the outer RNA strand acts both as phosphorylation acceptor substrate and as part of the ribozyme.

A series of tethered ribozymes was obtained by transcribing the outer RNA transcript from templates that appended one of several connector segments. There were eight tethered variants with connecting loops varying in length from 1 to 20 nts. For the comparison of the activities of the variants, APM-PAGE was used as a useful means which allows analysis of thiolated or thiophosphorylated RNA by the strong interaction between mercury and sulfur. ${ }^{5-7}$ The mobility of RNA that carries thiophosphate monoester is diminished, compared with non-thiophosphated one. This is the evidence of strong interaction between mercury and sulfur. Disulfides don't interact with the mercury in the gel matrix containing APM. ${ }^{6}$ Therefore, tethered variants of Kin. 46 were incubated with $\mathrm{ATP} \gamma \mathrm{S}$ and the products were separated by PAGE using gels that contained APM. The observed rate constants for the self-thiophosphorylation reaction were determined by measuring the initial velocity of the reaction at various ATP $\gamma \mathrm{S}$ concentration. The observed rate constants for the thiophosphorylation of the ribozymes are shown in Fig. 2. The tethered ribozyme with the shortest connection domain ( $1 \mathrm{nt}$ ) was barely active, and the values of $\mathrm{k}_{\text {cat }}$ increased with the length of the connection loop, with nearly full activity 
(A)

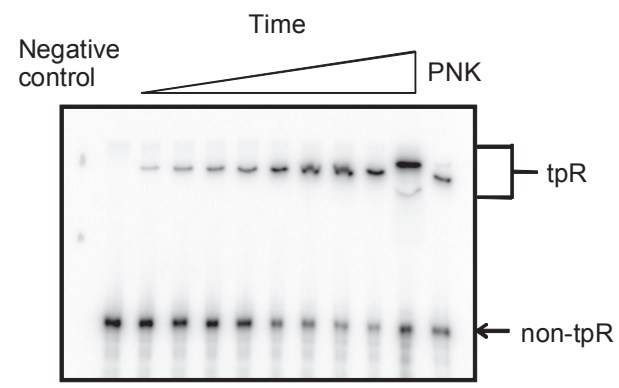

(B)

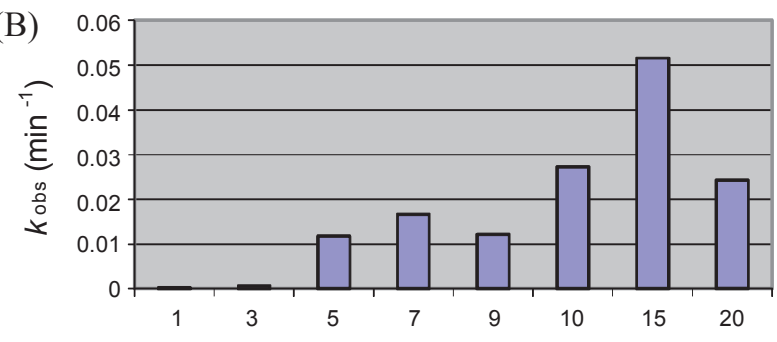

Numbers of nucleotides in connecting loop

Figure 2. Kinetic assay of the tethered ribozymes. (A) Autoradiogram for the self-thiophosphorylation of 15-nt tether ribozyme. The 15-nt tether ribozymes thiophosphorylated (tpR) with ATP $\gamma \mathrm{S}$ were separated from the nonthiophosphorylated ribozymes (non-tpR) with APMPAGE. PNK lane treated with polynucleotide kinase was also applied to normalize each lane. (B) Observed catalytic rate constant values for self-thiophosphorylation reaction for the tethered ribozymes according to the size of the loop connecting helices P3 and P4. Nucleotide sequences in connecting loop are as follows; 1 -nt, A; 3-nt, GGA; 5-nt, UUGGA; 7-nt, ACUUGGA; 9-nt, ACACUUGGA; 10-nt, AACACUUGGA; 15-nt, CAGAUAACACUUGGA; 20-nt, AAUAACAGAUAACACUUGGA.

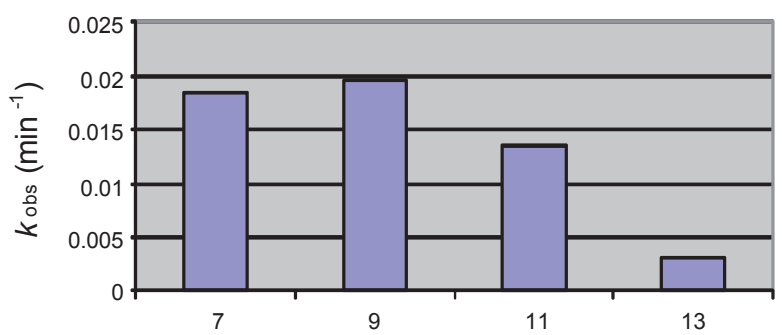

Numbers of nucleotides complementary to 15 -nt connector

Figure 3. Observed catalytic rate constant values for self-thiophosphorylation reaction with $\mathrm{ATP} \gamma \mathrm{S}$ for tethered ribozyme with the 15-nt connector by annealing several DNA oligonucleotides complementary to the tether. Nucleotide sequences complementary to 15-nt connecting loop are as follows; 7-nt, AGTGTTA; 9-nt, AAGTGTTAT; 11-nt, CAAGTGTTATC; 13-nt, CCAAGTGTTATCT.

recovered when the tether reached a length of $7 \mathrm{nts}$, compared to the nontethered ribozyme. ${ }^{7}$ A 7-nt connector is therefore long enough to accommodate the optimal spacing between the termini of $\mathrm{P} 3$ and $\mathrm{P} 4$. The explanation is that the longer tethers allows the ribozyme to relax into an active fold by simultaneously forming P3 and P4 in a manner that can assume productive orientations with respect to each other. Activity is maximal for the ribozyme with the 15 -nt connector, so this construct was chosen for further study.

Annealing a 13-nt DNA oligonucleotide complementary to the 15-nt tether reduced the observed catalytic rate by approximately seven-fold, demonstrating that an A-form helical connection between the termini of P3 and P4 is not tolerated (Fig. 3). Shorter oligonucleotides (11, 9 and $7 \mathrm{nts}$ ) had progressively less effect on catalysis, but they also did not remain bound in a native gel shift assay, and may have been excluded from the complex.

The dependence of activity on the linker length suggests that long-range distance constraints can aid in indicating an optimal distance between the distal termini of $\mathrm{P} 3$ and P4. The shortest tether that allowed most activity to be restored was $7 \mathrm{nts}$ in length. A tether of this length is capable of spanning $35-40 \AA$ if fully extended ( 5 - $6 \AA / \mathrm{nt}$ for flexible chain). ${ }^{8}$ Half of the normal activity was recovered for a 5-nt tether $(\sim 25-30 \AA)$. Assuming that neither connector is fully extended in the absence of a strong external stretching force, the connector segment can be viewed as a single-stranded molecular ruler indicating an optimal distance of $\sim 25-35 \AA$ between the distal termini of P3 and P4.

\section{Experimental Section}

Preparation of ribozymes. Ribozyme strands were synthesized by run-off transcription of PCR DNA templates using T7 RNA polymerase. ${ }^{32} \mathrm{P}$ internally labeled outer-RNA strand was synthesized by T7 RNA polymerase run-off transcription in the presence of $\left[\alpha-{ }^{32} \mathrm{P}\right]$ UTP. RNA transcripts were purified from $8 \%$ denaturing ( $7 \mathrm{M}$ urea) polyacrylamide gels using 90 $\mathrm{mM}$ Tris-borate $(\mathrm{pH} 8.3)$ and $2.5 \mathrm{mM}$ EDTA.

Kinetic assay of ribozyme. Rates of RNA-catalyzed thiophosphoryl transfer reaction were measured using internally ${ }^{32} \mathrm{P}$-labeled outer-RNA strand, synthesized as internally radiolabeled transcript using $\left[\alpha-{ }^{32} \mathrm{P}\right]$ UTP. Active complex was assembled using $1 \mu \mathrm{M}$ outer-RNA strand and $2 \mu \mathrm{M}$ inner-RNA strand of ribozyme ( $4 \mu \mathrm{M}$ single strand deoxyribonucleotide complementary to 15-nt connector was added for annealing analysis). Outer-RNA strand and inner-RNA strand were heated in $\mathrm{KCl} /$ Pipes buffer (200 mM KCl in $150 \mathrm{mM}$ Pipes-KOH, pH 7.0) at $90^{\circ} \mathrm{C}$ for $2 \mathrm{~min}$ and allowed to cool to RT $\left(\sim 21^{\circ} \mathrm{C}\right)$. TCEP (final concentration $10 \mathrm{mM}$ ) was added to maintain a reducing environment and to avoid disulfide bond formation between thiophosphorylated products. ${ }^{6,9}$ These were adjusted to a final concentration of $50 \mathrm{mM} \mathrm{MgCl}_{2}$ and preincubated for $15 \mathrm{~min}$ at RT. An aliquot was immediately removed to serve as zero time point. The thiophosphorylation reaction was initiated by addition of ATP $\gamma \mathrm{S}$ to $10 \mathrm{mM}$ at RT. Aliquots were removed at various times (2 min, $5 \mathrm{~min}, 8 \mathrm{~min}, 10 \mathrm{~min}, 110 \mathrm{~min}, 230 \mathrm{~min}, 340 \mathrm{~min}, 1300$ $\mathrm{min}$ and $1450 \mathrm{~min}$ ) and the reaction quenched on dry ice with $94 \%$ formamide, $30 \mathrm{mM}$ EDTA ( $\mathrm{pH} 8.0$ ) containing xylene cyanol and bromophenol blue. Thiophosphorylated products were separated from the unreacted nonthiophosphorylated strands by electrophoresis in APM $(100 \mu \mathrm{g} / \mathrm{mL}) 6 \%$ polyacylamide gel in $90 \mathrm{mM}$ Tris-borate $(\mathrm{pH} 8.3)$ and $2.5 \mathrm{mM}$ EDTA containing $7 \mathrm{M}$ urea. This takes advantage of the strong interaction between mercury and thiolate ions ${ }^{5}$ and has proven valuable for the selection and kinetic analysis of kinase ribozymes. ${ }^{6,9}$ 
Dried gels were exposed to storage phosphor screens and analyzed using ImageQuant software (Molecular Dynamics). The extent of thiophosphorylation was estimated by dividing the radioactivity in the product band (retained at the top of the APM layer) by the sum of reacted and unreacted bands. Internally radiolabeled outer-strand RNA was terminally thiophosphorylated by polynucleotide kinase (New England Biolabs) and ATP $\gamma$ S to serve as normalization control in determining the fraction reacted for each of the experimental above. The data were fit to a kinetic equation; The first-order rate of thiophosphorylation ( $k_{\text {obs }}$ : observed rate constant) was calculated by fitting to $\mathrm{f}_{\mathrm{t}}=\left(\mathrm{f}_{\infty}-\mathrm{f}_{0}\right)\left(1-\exp \left(-k_{\mathrm{obs}} \mathrm{t}\right)\right)$, where $\mathrm{f}_{\mathrm{t}}$ is the fraction normalized at time $t$.
Acknowledgments. I thank Prof. Donald H. Burke (University of Missouri) for his helpful support.

\section{References}

1. Lorsch, J. R.; Szostak, J. W. Nature 1994, 371, 31.

2. Lorsch, J. R.; Szostak, J. W. Biochemistry 1995, 34, 15315.

3. Cho, B.; Burke, D. H. Bull. Korean Chem. Soc. 2007, 28, 463.

4. Cho, B. Bull. Korean Chem. Soc. 2007, 28, 689.

5. Igloi, G. L. Biochemistry 1988, 27, 3842.

6. Rhee, S. S.; Burke, D. H. Analytical Biochem. 2004, 325, 137.

7. Cho, B.; Burke, D. H. RNA 2006, 12, 2118.

8. Williams, M.; Rouzina, I.; Wenner, J.; Gorelick, R.; Musier-Forsyth, K.; Bloomfield, V. Proc. Natl. Acad. Sci. 2001, 98, 6121.

9. Saran, D.; Nickens, D.; Burke, D. Biochemistry 2005, 44, 15007. 